\title{
Estudio Biométrico de la Arterias Femoral, Poplítea y Tibial Posterior en Relación a los Implantes de Stents
}

\author{
Biometric Study of the Femoral, Popliteal and Posterior Tibial Arteries in Relation to the Stents
}

\author{
Moreira da Costa Filho, E.",**; Avelino dos Santos, T. F."; Avelino dos Santos, F. T.; Honorato Pereira, V. H."; \\ Brandão Pitta, G. B.*; del Sol, M.***,***; Olave, E..** \& Sousa-Rodrigues, C. F.*****
}

COSTA FILHO, E. M.; DOS SANTOS, A. T. F.; DOS SANTOS, A. F. T.;PEREIRA, V. H. H.; PITTA, G. B. B.; DEL SOL, M.; OLAVE, E. \& SOUSA-RODRIGUES, C. F. Estudio biométrico de las arterias femoral, poplítea y tibial posterior en relación a los implantes de Stents. Int. J. Morphol., 32(2):652-655, 2014.

RESUMEN: Los aneurismas de la arteria poplítea son los más frecuentes entre los aneurismas periféricos, pudiendo ser tratados con stents a través de cirugía endovascular. Sin embargo, tales stents poseen el mismo diámetro en la parte proximal y distal, no siendo compatible con la anatomía vascular de ese segmento. El objetivo del presente estudio fue determinar la diferencia entre el diámetro del tercio distal de la arteria femoral y el diámetro de la arteria tibial posterior, además de obtener el diámetro de otras arterias del miembro inferior. Se utilizaron 28 cadáveres formolizados de individuos adultos, de ambos sexos, los que fueron disecados en la región correspondiente. Los diámetros registrados fueron los siguientes: en el tercio distal de la arteria femoral (inicio de la arteria poplítea), parte final de la arteria poplítea antes de su bifurcación en arterias tibial anterior y posterior, arterias tibial anterior y posterior (tronco tibiofibular). En el sexo masculino, desde el tercio distal de la arteria femoral hasta la arteria tibial posterior hubo una reducción del diámetro de $27,92 \%$ ( $\mathrm{p}<0,0001$ ). En el femenino, la reducción fue de 52,61\% ( $<$ que 0,0001). Al comparar el diámetro del tercio distal de la arteria femoral entre ambos sexos, se obtuvo una diferencia estadísticamente significativa ( $\mathrm{r}$ 0,0497), así como entre los diámetros de las arterias tibiales posteriores $(\mathrm{p}<0,0142)$. Estos datos son de importancia clínica para los procedimientos de cirugía endovascular, pudiendo reducir las complicaciones en tales procedimientos. Las endoprotésis deberán adaptarse a estas diferencias de diámetro.

PALABRAS CLAVE: Anatomía; Miembro inferior; Arterias.

\section{INTRODUCCIÓN}

La enfermedad arterial obstructiva periférica es la manifestación más común de la enfermedad arteroesclerótica sistémica (Pinto \& Mandil, 2005). Se ha señalado que el $16 \%$ de la población de más de 55 años sufre de esta enfermedad (Medeiros \& Gaspar, 2006).

Actualmente, el tratamiento de las enfermedades vasculares periféricas es una de las áreas de la medicina con mayor expansión y existe hoy, además del tratamiento con cirugía vascular tradicional, la cirugía endovascular que a través de los stents y de la angioplastia, buscan mejorar el éxito del procedimiento. Con el creciente desarrollo tecnológico y el avance de las investigaciones para encontrar un reemplazo vascular ideal, el uso de stents se ha tornado una opción terapéutica promisoria (Lobato, 2006; França \& Pereira, 2008) y es utilizado para tratar patologías vasculares del miembro inferior.

Se sabe que los aneurismas de la arteria poplítea (AAP) son los más frecuentes dentro de los aneurismas periféricos, correspondiendo de 70 a $80 \%$ de los casos (Antonello et $a l ., 2005)$ y la enfermedad arteroesclerótica constituye la etiologia más común de los aneurismas poplíteos, aunque, la sífilis, los traumas y enfermedad micótica sean relatados también, como factor causal (Miranda, 1999; Oliveira et al., 2005). Así, una de las formas que se tiene para corregir esos AAP es a través de stents, idealmente aquellos que se adapten a los diámetros proximal y distal de tal segmento.

* Universidade Estadual de Ciencias da Saude de Alagoas, Brasil.

** Programa Iniciación Científica, FAPEAL, Curso de Medicina, Brasil.

*** Facultad de Medicina, Universidad de La Frontera, Chile.

***** Centro de Investigación en Ciencias Biomédicas Universidad Autónoma de Chile, Chile.

****** Universidade Estadual de Ciencias da Saude de Alagoas, Universidade Federal de Alagoas, Brasil. 
Marín et al.(1994) fueron los primeros en realizar el reparo endovascular del AAP. Después de eso, las tasas de permeabilidad aumentaron de forma significativa, sin embargo esa técnica tiene también desventajas, teóricamente, en cuanto a la región anatómica, porque se localiza en la región posterior de la rodilla y los stents no se adaptan de la mejor forma al diámetro proximal y distal de la arteria poplítea. De este modo, la prótesis implantada (stent) es sometida a un constante stress físico y al riesgo de oclusión (Tomazinho et al., 2008).

Se debe conocer bien el promedio del diámetro de las artérias para la utilización de stents, por ejemplo, en el tratamiento de los aneurismas. Los stents deben adaptarse a los diámetros arteriales y para ser producidos se hace necesario conocer el promedio de los diámetros arteriales en cada parte del cuerpo.

Actualmente, para el tratamiento endovascular de los AAP, se utilizan stents con diámetros iguales, tomándose como referencia el lumen de la porción proximal de la arteria poplítea e ignorando la porción distal de ésta. Esto trae algunos inconvenientes, como la dificultad técnica debido a la desproporción entre los diámetros de los segmentos arteriales proximal (arteria poplítea) y distal (arteria tibial posterior), culminando con dificultad de adaptación del diámetro distal del stent al diámetro proximal de la arteria poplítea.

Así, el conocimiento de la diferencia entre el diámetro del tercio distal de la arteria femoral y el diámetro de la arteria tibial posterior puede ayudar en las investigaciones tecnológicas para producir stents cónicos que se puedan adaptar al segmento poplíteo y a la arteria tibial posterior.

\section{MATERIAL Y MÉTODO}

El estudio se realizó en 56 miembros inferiores de 28 cadáveres humanos, fijados en formaldehido $10 \%$, adultos, de ambos sexos, siendo 20 de sexo masculino y 8 del femenino. Posterior a la disección y con la ayuda de un caliper digital Gehaka (de 0- $300 \mathrm{~mm}$, resolución de $0,01 \mathrm{~mm}$ ) se registraron los diámetros de la arteria femoral a nivel de su tercio distal (en el hiato de los aductores - inicio de la arteria poplítea), diámetro de la parte final de la arteria poplítea (cuando ésta se divide en arterias tibial anterior y tibial posterior), diámetros de la arterias tibial anterior y tibial posterior antes de ésta emitir la arteria fibular y el de esta última.
Para la variable diámetro arterial se calcularon los promedios, el intervalo de confianza y la desviación standard, utilizando para ello el programa BioEstat 5.0, con la ayuda del producto Microsoft Excel 2007. El valor de alfa fue definido en 0,05 (5\%) y para la comparación entre sexos, se utilizo el test de Student, cuyas diferencias fueron consideradas significativas cuando $\mathrm{p}<0,05$.

\section{RESULTADOS}

El diámetro del tercio distal de la arteria femoral (inicio de la arteria poplítea) fue en promedio de 4,2 \pm $0,7 \mathrm{~mm}$ en el sexo masculino y de 4,63 $\pm 0,57 \mathrm{~mm}$ en el femenino. Al comparar los diámetros entre sexos se obtuvo una diferencia estadísticamente significativa ( $\mathrm{p}=$ 0,0497).

El diámetro de la arteria tibial posterior tuvo un promedio de $3,03 \pm 0,72 \mathrm{~mm}$ en el sexo masculino y de $2,43 \pm 0,58 \mathrm{~mm}$ en el femenino. Al comparar entre sexos la diferencia obtenida fue estadísticamente significativa $(\mathrm{p}=0,0142)$.

A partir de esto, observando las diferencias entre sexos, se analizó el porcentaje de reducción del diámetro del tercio distal de la arteria femoral (inicio arteria poplítea) en relación al diámetro de la arteria tibial posterior en cada sexo.

En el sexo masculino hubo una reducción de los diámetros de $27,92 \%$, o sea, la diferencia entre los diámetros de las arterias mencionadas fue estadísticamente significativa $(p<0,0001)$. En el sexo femenino, la reducción fue de $52,61 \%$ y la diferencia entre los diámetros también fue significativa $(\mathrm{p}<0,0001)$.

En relación al diámetro de la parte final de la arteria poplítea (cuando ésta se divide en arterias tibial anterior y tibial posterior), su promedio fue de $3,58 \mathrm{~mm}$. Con respecto a los diámetros de la arteria fibular $(2,1 \pm 0,3$ $\mathrm{mm}$ ), en 42 casos fueron menores que los de otras arterias registradas. En 13 casos de cadáveres diferentes, el diámetro de la arteria fue mayor que el de la arteria tibial anterior $(2,6 \mathrm{~mm})$ y el de la arteria tibial posterior. En un caso no fue posible registrar el diámetro de este vaso.

En 5 miembros de cadáveres diferentes no fue posible localizar y disecar a la arteria tibial anterior, probablemente por variación anatómica o porque estaba lacerada. 


\section{DISCUSION}

El conocimiento de los diámetros de las arterias del miembro inferior en cadáveres es una área poco estudiada y uno de los mayores problemas actuales en países desarrollados son las enfermedades circulatorias, así como su prevención y tratamiento, ya que éstas aparecen como una importante causa de morbilidad y mortalidad (Jacobovicz et al., 2004).

Bajo esa perspectiva, la cirugía endovascular con utilización de stents, ha ganado un importante espacio en el tratamiento de varias enfermedades vasculares que antes eran tratadas a través de cirugía convencional (Antonello et al.). Una de las preocupaciones en el desarrollo de stents para tratar las diversas enfermedades vasculares es la adaptación del stent al diámetro de los vasos, por lo que es necesario que sean producidos con diámetros apropiados, por lo adquiere importancia poseer un conocimiento adecuado de los diámetros de los vasos del cuerpo ( Moore \& Dalley, 2011).

Los aneurismas de la arteria poplítea son los más frecuentes dentro de los aneurismas periféricos, 70-80\% de los casos (Antonello et al.) y la enfermedad arteroesclerótica constituye la etiología más común de los aneurismas poplíteos, aunque también se ha mencionado la sífilis, los traumas y las infecciones por hongos, como factor causal (Oliveira et al.).
Una forma de tratar los AAP es con stents, sin embargo, los stents utilizados poseen un diámetro proximal (que se fija al tercio distal de la arteria femoral y que corresponde al inicio de la arteria poplítea) y un diámetro distal (en la arteria tibial posterior) del mismo tamaño. Debido a la desproporción entre los diámetros de los segmentos arteriales proximales y distales (inicio de la arteria poplítea y arteria tibial posterior, respectivamente), se pueden presentar inconvenientes técnicos por la dificultad de adaptación del diámetro distal del stent al diámetro proximal de la arteria tibial posterior.

Esta investigación deja claro que hay una disminución significativa del diámetro entre los distintos vasos. Así, comparar el diámetro del tercio distal de la arteria femoral con el diámetro de la artera tibial posterior, se verifica que esta disminución es mayor en indivíduos de sexo femenino.

Los resultados presentados pueden ayudar a investigaciones sobre el desarrollo y producción de stents, que puedan adaptarse a los diferentes diámetros de las arterias del miembro inferior, inclusive stents cónicos que se adapten al segmento poplíteo-tibial posterior, pudiendo tratar adecuadamente los AAP por cirugía endovascular.

COSTA FILHO, E. M.; DOS SANTOS, A. T. F.; DOS SANTOS, A. F. T.;PEREIRA, V. H. H.; PITTA, G. B. B.; DEL SOL, M.; OLAVE, E. \& SOUSA-RODRIGUES, C. F. Biometric study of the femoral, popliteal and posterior tibial arteries in relation to the Stents. Int. J. Morphol., 32(2):652-655, 2014.

SUMMARY: The popliteal artery aneurysms are the most common among peripheral aneurysms and can be treated with stents through endovascular surgery. However, these stents have the same diameter at the proximal and distal part, not being compatible with the vascular anatomy of that segment. The aim of this study was determine the difference between the diameter of the distal third of the femoral artery and the diameter of the posterior tibial artery, and to obtain the diameter of others arteries of the lower limb. We study 28 formalized cadavers of adult individuals of both sexes, which were dissected in the corresponding region. The following arterial diameters were recorded: the distal third of the femoral artery, distal part of the popliteal artery, anterior tibial artery and posterior tibial artery. In males from the distal third of the femoral artery to the posterior tibial artery there was a narrowing of $27.92 \%$ ( $<<0.0001$ ). In women , the reduction was $52.61 \%(\mathrm{p}<0.0001)$. By comparing the diameter of the distal third of the femoral artery between the sexes, a statistically significant difference was obtained ( $\mathrm{p}<0.0497)$, as well as between the posterior tibial artery diameters $(\mathrm{p}<0.0142)$. These data are of clinical importance for endovascular surgery procedures and may reduce complications in such procedures. Stents must adapt to these differences in diameter.

KEY WORDS: Anatomy, Lower limb, Arteries.

\section{REFERENCIAS BIBLIOGRÁFICAS}

Antonello, M.; Frigatti, P.; Battocchio, P.; Lepidi, S.; Cognolato, D.; Dall'Antonia, A.; Stramanà, R.; Deriu, G.P. \& Grego, F. Open repair versus endovascular treatment for asymptomatic popliteal artery aneurysm: results of a prospective randomized study. J. Vasc. Surg.,42:185-93, 2005. 
COSTA FILHO, E. M.; DOS SANTOS, A. T. F.; DOS SANTOS, A. F. T.;PEREIRA, V. H. H.; PITTA, G. B. B.; DEL SOL, M.; OLAVE, E. \& SOUSA-RODRIGUES, C. F. Estudio biométrico de las arterias femoral, poplítea y tibial posterior en relación a los implantes de Stents. Int. J. Morphol., 32(2):652-655, 2014.

França, L. H. G. \& Pereira, A.H. Atualização sobre endopróteses vasculares (stents): dos estudos experimentais à prática clínica. J. Vasc. Bras., 7(4):35163, 2008.

Jacobovicz , C.; Timi, J. R., França, L. H. G.; Stahlke Jr., H. J. \& Nakahara, J. Avaliação do eco-Doppler na predição da necessidade de arteriografia do território aorto-ilíaco em pacientes submetidos a revascularização arterial infra-inguinal. J. Vasc. Br., 3(1):5-12, 2004.

Lobato, A.C. Stents Vasculares. In: Lobato, A.C. Cirurgia Endovascular. São Paulo: Instituto de Cirurgia Vascular e Endovascular de São Paulo, 2006. pp. 83-96.

Marin, M.; Veith, F.J.; Panetta, T.F.; Cynamon, J.; Bakal, C. W.; Suggs, W. D.; Wengerter, K. R.; Baronè, H. D.; Schonholz, C. \& Parodi, J.C. Transfemoral endoluminal stented graft repair of a popliteal artery aneurysm. $J$. Vasc. Surg., 19:754-7, 1994.

Medeiros, C. A. F. \& Gaspar, R. J. Correção endovascular do aneurisma de artéria poplítea bilateral. J. Vasc. Bras., 5 (4):303-7, 2006.

Miranda, Jr. F. Aneurisma da artéria poplítea: risco do não diagnóstico. Rev. Soc. Cardiol. Estado de São Paulo, 9:873-8, 1999.

Moore, K. L.; Agur, A. M. R. \& Dalley, A. F. Anatomia orientada para a Clínica. $6^{\mathrm{a}}$ ed. Rio de Janeiro, Guanabara Koogan, 2011.

Oliveira, R. S. M. ; Ferreira, D. A.; Terra Júnior, J. A.; Lenza, R. M.; de Assis Filho, A. C. \& Nunes, W. Ruptura de aneurisma da artéria poplítea: relato de caso e revisão da literatura dos últimos 50 anos. J. Vasc. Bras. 4(1):105$10,2005$.

Pinto, D. M. \& Mandil, A. Claudicação Intermitente: do Tratamento Clínico ao Intervencionista. Rev. Bras. Cardiol. Inv., 13(4): 261-9, 2005.

Thomazinho, F.; Silvestre, J. M. S.; Sardinha, W. E.; Motta, F., Perozin, I. Sch. \& Morais Filho, D.Tratamento Endovascular de Aneurisma de Artéria Poplítea. J. Vasc. Bras., 7(1):38-43, 2008.
Dirección para correspondencia:

Célio Fernando de Sousa Rodrigues.

Av. Álvaro Otacílio, 6742.

Edf. Saint Thomaz, apt 201, Jatiúca, 57036-850.

Maceió - AL.

BRASIL

Email: celiofernando@yahoo.com.br

Recibido: 12-01-2014

Aceptado: 25-03-2014 